\title{
Tratamiento Social de las Diferencias, Teorias Infantiles y Narrativas
}

\author{
Rosana Maneiro' \\ Mercedes Minnicelli' \\ 'Universidad Nacional de Mar Del Plata (UNMDP), Buenos Aires - Argentina
}

RESUMEN - Tratamiento Social de las Diferencias, Teorías Infantiles y Narrativas. En este escrito nos ocuparemos del tratamiento social de las diferencias y sus resonancias singulares en tres direcciones: a) la importancia en el devenir de la sexualidad infantil del encuentro con la diferencia entre los cuerpos como percepción clave en la configuración de teorías sexuales infantiles y sus repercusiones cuando las mismas no cuentan con soporte colectivo de tramitación; b) la tendencia a la patologización y medicalización de la infancia ante la falta de escenarios de consideración de dichas teorías; c) alternativas clínico sociales para intentar resistir a la devastación subjetiva de las nuevas generaciones.

Palabras-clave: Medicalización de la Infancia. Teorías Sexuales Infantiles. Género y Sexuación. Recuperación de Narrativas.

ABSTRACT - Social Treatment of Differences, Childhood Theories and Narrative. In this paper we look at the social treatment of the differences and unique resonances in three directions: a) The importance in the evolution of infantile sexuality of the encounter with the difference between the bodies as key in shaping perceptions of infantile sexual theories and their impact when they do not have collective processing support; $b$ ) The trend of pathologizing and medicalization of childhood in the absence of such scenarios considering theories; c) Clinical social alternatives to try to resist the devastation of future generations subjective.

Keywords: Medicalization of Infancy. Infantile Sexual Theories. Gender and Sexuation. Recovery of Narrative. Education. 
Tratamiento Social de las Diferencias, Teorias Infantiles y Narrativas

En este escrito nos ocuparemos del tratamiento social de las diferencias y sus resonancias singulares en tres direcciones:

a) la importancia en el devenir de la sexualidad infantil del encuentro con la diferencia entre los cuerpos como percepción clave en la configuración de teorías sexuales infantiles y sus repercusiones cuando las mismas no cuentan con soporte colectivo de tramitación;

b) la tendencia a la patologización y medicalización de la infancia ante la falta de escenarios de consideración de dichas teorías;

c) alternativas clínico sociales para intentar resistir a la devastación subjetiva de las nuevas generaciones por el recupero de narrativas que den lugar al despliegue y configuración de lo imaginario como instancia psíquica de anudamiento de lo simbólico, borde a lo real de la experiencia, el devenir y despliegue de la sexualidad.

Consideramos necesario recuperar debates académicos y psicoanalíticos respecto de la importancia que presenta en el devenir de la subjetividad/sexualidad infantil, el encuentro con la diferencia entre los cuerpos y la producción de teorías sexuales infantiles como respuesta ante el enigma que la percepción de la diferencia provoca al sujeto en configuración.

Sus efectos en la configuración subjetiva, sus formas de tramitación serán cimientos de las tres clásicas posiciones que el psicoanálisis plantea respecto de la configuración de estructuras clínicas (neurosis, psicosis, perversión). Categorías en revisión respecto de su estabilidad permanente; su movilidad estructural; sus bordes, sus fracturas.

En Tres ensayos... Freud presentó una noción de compleja aceptación en nuestros días por la puesta en juego de significantes cuyas resonancias contrastan, confrontan con la noción de niño rousseuniana: pequeños perversos polimorfos.

Por esta denominación, “[...] lejos de realizar un catálogo de prácticas perversas” avanzó contra “[...] cualquier naturalización del encuentro sexual” (Cevasco, 2010, p. 71).

Podemos recordar que allí expresa:

La diferencia más honda entre la vida sexual de los antiguos y la nuestra reside, acaso, en el hecho de que ellos ponían el acento en la pulsión misma, mientras que nosotros lo ponemos sobre su objeto. Ellos celebraban la pulsión y estaban dispuestos a ennoblecer con ella incluso a un objeto inferior, mientras que nosotros menospreciamos el quehacer pulsional mismo y lo disculpamos sólo por las excelencias del objeto (Freud, 1908a, p. 137).

Freud (1908a; 1908b; 1914; 1915; 1923; 1924; 1925) en diferentes momentos de su producción científica se ocupó de la importancia tanto de la sexualidad infantil como de la construcción de teorías sexuales que los niños y niñas construyen en el devenir de su configuración sub- 
jetiva. Les otorgó un valor estructural y estructurante en la lógica subjetiva en tiempos de su constitución (Minnicelli, 2010).

Para un infantil sujeto el descubrimiento de las diferencias, implica la experiencia del desencuentro estructural y estructurante en la relación central de objeto del hablante, puesto que "la relación central de objeto, la que es dinámicamente creadora, es la de la falta" (Lacan, 1994, p. 54), resultando móvil para la renovación de preguntas ontológicas respecto de Dios, de la muerte, del nacimiento y del sexo.

La producción de narrativas a partir de dichas teorías, resiste a la contrastación de cualquier supuesta verdad científica, resiste a la prueba de realidad; de allí su valor en la configuración de lo imaginario, en la vertiente estructural y estructurante. Hito subjetivo clave en la configuración del enlace por lo imaginario, de lo simbólico bordeando lo real de la experiencia. Real como agujero; real que se reedita una y otra vez, ante el reconocimiento de la diferencia en la hiancia presencia-ausencia; imposibilidad del sexo; imposibilidad de representación psíquica de la muerte.

Cuando lo real no encuentra marco que permita por lo imaginario configurar bordes, es decir, cuando un niño no cuenta con las condiciones de posibilidad para su emergencia, la confrontación con lo real de la experiencia deja al psiquismo incipiente en un estado clínicamente comprobado de desorden subjetivo alienante. Tal el caso de aquellos niños y niñas cuyos cuerpos son expuestos al sexo, como objeto de goce de mayores.

Jacques Lacan resalta el valor de las teorías sexuales infantiles, ubicándolas en relación al mito:

En suma, para centrar el valor exacto de las llamadas teorías sexuales infantiles hemos de referirnos a la noción de mito. Lo que se llama un mito se presenta como un relato. Pueden decirse muchas cosas sobre ese relato, por ejemplo que tiene algo de atemporal puede tratar de definirse su estructura en relación con los lugares que define por que muestra ciertas circunstancias que no están sometidas en absoluto a la invención subjetiva. También indicar el problema planteado por el carácter de ficción que el mito tiene en conjunto. Pero esta ficción representa una estabilidad que implica que cualquier modificación supone al mismo tiempo alguna otra sugiriendo, invariablemente, la noción de estructura. Por otra parte esta ficción mantiene una singular relación con algo que siempre se encuentra detrás implicado, se trata de la verdad. (Lacan, 1994, p. 251).

Pablo Peusner (2006) sostiene:

Es el momento de los por qué o, como decía Freud, el momento de la teorización sexual infantil. Lacan propone una máxima tensión entre el niño como sujeto y la batería significante como lo Otro. Esto es así porque Lacan sostie- 
Tratamiento Social de las Diferencias, Teorias Infantiles y Narrativas

ne que la pregunta, si bien es dirigida a los padres, surge cuando el niño tiene cierto dominio de la estructura significante (p. 146).

Vale destacar que, si bien la pregunta es dirigida a los padres, surge cuando el niño logra cierto dominio de la estructura significante. La actividad del preguntar indica que se trata de dos territorios topológicos: el campo del Otro - que recibe la pregunta - y el campo del infantil sujeto - quien la formula.

Bien sabemos que el Otro no está en condiciones de dar una respuesta que satisfaga la demanda pulsional que motoriza la investigación sexual infantil. Justamente de eso se trata. El niño des-cubre el todo saber de los mayores; des-cubre la imposibilidad de la respuesta certera y en esa falta de respuesta cierta, configura sus propias teorías imaginarias que las contrasta con las respuestas de los mayores.

Por Freud, desde 1908, estamos alertados acerca de que los significantes muerte y sexo, son nombres del agujero en la estructura simbólica; precisamente por ello, es posible considerar que no siendo la sexualidad humana meramente reproductiva ni natural, son posibles modificaciones jurídicas y políticas que alteran las estructuras de parentesco, los modelos de la filiación y las formas de la organización familiar según las épocas y las construcciones de los imaginarios admitidos o configurados como prohibidos. Las diferencias culturales, se asentarán en lo que para cada una de ellas es aceptado o rechazado por el colectivo social. A pesar de ello, cada sujeto siempre tiene margen para mantener con los discursos de las hegemonías y sus categorías sociales, una relación de proximidad, de rechazo o de distancia.

Nos interesa dar lugar al tratamiento social de las diferencias, en su posibilidad de pensar sus vínculos centrales con la educación.

Llegados a este punto, debemos revisar ciertas categorías sociales y políticas desde el marco epistémico que nos aporta el psicoanálisis, cuando:

Las discusiones de los últimos años sobre la diferencia sexual ha ocupado un primer plano como factor de la política, de la epistemología, de los movimientos feministas y de liberación sexual, nosotros, los psicoanalistas, debemos hacer un esfuerzo para situarnos en el debate de manera clara, porque hay muchos conceptos que van y vienen, y la confusión está servida (Cevasco, 2010, p. 42).

Enunciar diferencia de los sexos; género y sexuación ${ }^{1}$ merecen de aclaraciones, especialmente si nos interesa dejar abiertos sus vínculos no sólo con la clínica, sino también con la educación.

Siguiendo a Rithee Cevasco (2010), consideraremos por sexolo que denota a la referencia puramente anatómica (salvo casos excepcionales), distinguiendo entre macho y hembra. Quedarnos con la percepción de esta diferencia donde, desde Freud, se asientan las bases y funda- 
mentos de las teorías sexuales infantiles, sería detenerse en un tiempo parcial de la teorización clínica del psicoanálisis. La percepción de la diferencia y la denegación de la falta, va más allá del cuento sobre tener o no tener pene. Se trata de la configuración de lo imaginario, de la constitución fantasmática que al enunciar Ya sé que... pero aún así.... ilustra la presencia del velo que cubre lo real de la falta. Lo real se viste y se tolera si hay formas sociales de re-cubrirlo, siendo las repercusiones de lo social siempre singulares.

Macho y hembra no deben confundirse con masculino y femenino. Freud presentó estas distinciones y separó con ellas a la diferencia sexual anatómica respecto de las posiciones activas y pasivas, en sus alternancias posibles y en sus estancamientos según fuera el sujeto en cuestión.

Esta distinción es renombrada por campos epistémicos no estrictamente psicoanalíticos. Es preciso entonces avanzar en las diferenciaciones para no alentar malos entendidos.

Designar el género, es referir a una construcción social de lo que es masculino y femenino, aquello que se construye según los ideales, los modelos sociales, las normas, etc; lo que se determina debe ser un hombre y una mujer, haciendo de la masculinidad y la femineidad algo más que lo simplemente anatómico, sujeto a configuraciones histórico sociales, culturales, religiosas, jurídicas y políticas.

Ahora bien, la noción de género y las construcciones sociales para definir lo que es válido como masculino y femenino, no se corresponden con las posiciones femenino y masculino freudianas.

Lacan diferenciará a ambas nociones de lo que denominó sexuación, es decir, una especificidad de las relaciones del sujeto con su goce (Cevasco, 2010, p. 46). Será entonces el género aquello que se vincule de manera más directa con las formaciones psíquicas que llamamos las identificaciones (Cevasco, 2010, p. 46).

Las teorías sexuales infantiles no responden a una construcción social, son singulares; tampoco responden a cuestiones de género y, si bien estarían vinculadas a la percepción de las diferencias sexuales anatómicas, escapan a ella. Este punto es sustantivo cuando no se trata de una cuestión de información o de conocimiento. Tampoco se trata de ofrecer modelos de identificación proclives a unas u otras construcciones sociales.

Las teorías sexuales infantiles, desde Freud, refieren a las bases de la configuración de lo imaginario a partir del encuentro con lo real de la experiencia, dando lugar a su posterior desarrollo sobre el complejo de castración en tanto operación psíquica compleja.

\section{Notas sobre los Enigmas de Juanito}

Freud construye los fundamentos de las teorías sexuales infantiles a partir de los enigmas de Juanito. Según se analice la posición del pa- 
Tratamiento Social de las Diferencias, Teorias Infantiles y Narrativas

dre y de la madre de aquel niño paradigmático, será posible identificar dos vías de enigmas: una que pertenece a los padres - campo del Otro y - otra, propia de Juanito - campo del infantil sujeto- que interrogando a los adultos, configura sus singulares respuestas.

Juanito se interroga a partir del descubrimiento de algo que no entiende y escapa a su control. Juanito se encuentra ante lo Real y sus teorías resultan las respuestas imaginarias que el infantil sujeto se da ante lo inexplicable. Juanito se enfrenta a los primeros enigmas de la vida y, ante ellos, tener o no tener es la cuestión.

En las primeras notas de las cartas que el padre de Juanito le envía a Freud, escribe que no les resulta agradable que su hijo empiece a plantearle enigmas y, podríamos agregar, esto no ha variado a pesar de los tiempos. A los mayores incomodan las preguntas de los niños, quienes nos enfrentan a los propios enigmas y a las propias teorías, ficciones que construimos para tolerar lo real.

Si algo ha caracterizado a los tiempos modernos, ha sido la convicción en la posibilidad humana de resolver esos enigmas que movilizaron a nuestros antepasados; convicción sobre que es posible - por la ciencia racional - hallar respuestas a aquello que, desde tiempos remotos, contó con ficciones de los más diversos órdenes: míticas, religiosas, filosóficas. Especialmente en los últimos siglos, la apuesta a la ciencia y a la razón como garante de la verdad, con la tecnología a su servicio, ha sido la clave para la posibilidad de una vida con los enigmas ya resueltos. Sin embargo, cada nuevo niño nos enfrenta a la imposibilidad y es preciso recordar que cada nuevo niño deberá atravesar por la posibilidad de encontrarse ante esos enigmas y de hallar el modo singular de respuesta, siempre ficcional.

Cuál es la verdad de los asuntos humanos, es un tema que no se agota - y esperemos nunca lo haga. Es porque aún existe un orden del enigma, testimonio de la falta; testimonio de lo que resta por decirse, que el deseo se mantiene vivo en un mundo que pugna por anularlo. Tendencia que pregona la ilusión del encuentro con la verdad y, el psicoanálisis nos enseña que todo encuentro, es y será siempre un encuentro fallido porque lo real no acude a la cita que ninguna teoría, ningún discurso le otorgue, por tanto, tratándose de la tendencia a la ilusión, nos debemos dirigir hacia la compleja lógica de las creencias, de la mistificación que sostiene dicha ficción en término de alentar la posibilidad de que existe Un Saber y que el mismo es pasible de ser portado por alguien.

En el Seminario 4, en la clase del 27 de marzo de 1957, Lacan plantea que:

Las llamadas teorías infantiles, es decir, la actividad de investigación propia del niño en lo referente a la realidad sexual, responde a una necesidad bien distinta que eso que llamamos, además incorrectamente, con una noción vaga, la actividad intelectual, cuyo carácter superestruc- 
tural admite de forma más o menos implícita el fondo de creencia que constituye el orden de la conciencia común (1994, p. 251).

Los enigmas a los cuales se enfrenta Juanito tanto como cualquier niño que llega a este mundo y, llegado el momento se enfrenta al interés por comenzar a descifrarlo, difiere de la actividad intelectual y conciente al comprometer al cuerpo en su conjunto y a toda actividad del infantil sujeto. Dirá Lacan (1994) que:

Engloba toda la actividad del sujeto y motiva todo lo que podemos llamar sus temas afectivos, es decir que dirige los afectos y las afecciones del sujeto de acuerdo con líneas de imágenes maestras [...] corresponde a toda una serie de efectuaciones, en el sentido más amplio, que se manifiestan por medio de acciones irreductibles a fines utilitarios (p. 251).

Las teorías sexuales infantiles carecen de fin utilitario. Quiebran la lógica de medio para un fin al representar un fin en sí mismo. Los infantiles sujetos, en el mejor de los casos, no pueden no pasar por la propia experiencia de verse afectados y sentir en el cuerpo la movilización de los afectos que el encuentro con lo real les provoca, ante lo cual, se renuevan las preguntas que la racionalidad psico-científica ha pretendido compensar o bien con información científica, o bien acallar con psicofármacos.

Sin embargo, las teorías sexuales infantiles resisten a ello y nos exponen que se trata de un tiempo sustantivo en la operación simbólica de escritura del infantil sujeto en la legalidad de la cultura, que no es otra que la ley simbólica que el discurso vehiculiza. En sí misma, en su estructura la ley plantea una zona de vacancia que no puede cubrirse sino es dejando siempre un resto in-asimilable, la narrativa presente en relatos míticos y fantásticos resulta aliada del lenguaje infantil.

Una clínica que se permite ubicar al psicoanálisis en otra escena, resultará diferencial para el tratamiento social de las diferencias en la medida que ese resto, eso in-asimilable pueda tener su lugar y ser bordeado, respecto de aquel tratamiento social de las diferencias que pretenda anularlo.

En esta última dirección ubicamos la clausura propuesta por la psico-farmacología que anula los enigmas que todo niño con dificultades en su desarrollo nos provoca. Cualquier tratamiento social de las diferencias sostenido al modo de las teorías sexuales infantiles, que desmienten las diferencias, sólo provocará sumisión, sometimiento y violencia. Dar lugar, por el trato discursivo y efectivo de tolerancia a las diferencias, si bien será más complejo - implicando renuncias a cualquier saber unificado - permitirá - en el mejor de los casos - la emergencia de otros dichos y posibilidades para nuevas significaciones.

Esta posición no es sin consecuencias para las nuevas generaciones y, sus manifestaciones hiperkinéticas y dis-atentas nos advierten 
Tratamiento Social de las Diferencias, Teorias Infantiles y Narrativas

respecto de la importancia de volver a ubicar lo enigmático en un lugar de privilegio: lo in-asimilable de la educación.

Dar lugar a los interrogantes, permitir preguntas y hallarse sinrespuestas resultan enlaces ficcionales que nos enfrentan a concordancias y discordancias inter-generacionales; no debemos olvidar que fueron preguntas ontológicas las que dieron nacimiento a la filosofía. įué dice un niño con su hiper-actividad? ¿Qué captura su atención, la cual leemos como dis-atención? Enigmas de los pequeños que nos enfrentan como mayores a los propios enigmas; a la propia posición subjetiva ante lo real de la experiencia. Preguntas que, de no darse lugar, alientan la perpetuación en la denegación por la desmentida, como formación psíquica para dar cobertura al descubrimiento de la falta en el Otro (denegada).

\section{Psicofármacos para no Preguntar, Remedios para no Soñar}

Tal como afirma Minnicelli (2010):

[...] se ha expropiado a las nuevas generaciones la propia experiencia ante la angustia, producto de la renovación de las preguntas ontológicas sobre el nacimiento, el sexo y la muerte, sustituyéndolas por explicaciones basadas en informaciones científica, frente al cual las "teorías sexuales infantiles" [...] oponen resistencia a ser domeñadas por la vía de la educación sexual (p. 33).

Helene L’Heuillet (2008) dirá:

[...] El niño no es ni una abstracción, ni un juguete: él es siempre "niño de", ligado a una filiación - más allá de soportarla fácilmente o no -. Por eso es que los psicoanalistas no pueden suscribir la idea de criar niños sólo con amor y cuidados. Porque nadie puede vivir sin palabra, la "necesidad" del niño es también una necesidad de lo simbólico (p. 105).

La infancia no es una época idílica y sin problemas con las que fantaseamos los adultos ${ }^{2}$, es un período de grandes convulsiones, de pasiones intensas, de miedos, incertidumbres. Es una época de crecimiento, de adquisiciones nuevas, en la que el soporte de los otros es fundamental. El psiquismo se estructura en una sociedad determinada y en una historia colectiva. Es decir, que nuestra historia es siempre a la vez una Otra historia. Concepto fundamental para recuperar la educación entendiendo que toda transmisión será fallida.

Al decir de Beatriz Janin (2011):

Ellos van construyendo soportes éticos desde la cuna. Y la construcción misma de los deseos como recorrido, como búsqueda, está marcada por la posibilidad del adulto de erotizar con ternura, sin dejar al otro en un estado de ex- 
citación permanente, de narcisizar reconociendo diferencias, de transmitir prohibiciones sin esgrimir funcionamientos autoritarios (p. 36-37).

Tema planteado por Sandor Ferenczi (1932) cuando estableció la diferencia entre el lenguaje infantil y el lenguaje adulto, lenguaje de la ternura y lenguaje de la pasión, entre el infantil sujeto y lo infantil del sujeto...

Mirtha Benítez y Ariel Pernicone (2010) sostendrán que el tratamiento posible del discurso estará en la relación a la situación que cada niño mantenga con el lenguaje según: el lugar que ha tenido en el deseo del Otro, como ha sido hablado por el Otro y el momento lógico de su constitución subjetiva.

En este marco teórico-clínico las conceptualizaciones sobre la importancia constitutiva y constituyente para el devenir de procesos simbólico-imaginarios, se concentra en torno a la expresión del hito subjetivo que las teorías sexuales infantiles representan.

Las formas de tramitación de dichas teorías, serán diferentes en la singularidad del caso. Tal como antes desarrollamos, resultan respuestas imaginarias ante el encuentro con las diferencias y, a partir de allí, con lo inexplicable para los infantiles sujetos respecto del nacimiento, Dios, la muerte y el sexo. Mediante este concepto teórico-clínico es posible considerar el enlace entre lo singular y sus efectos colectivos (según que se ofrezcan a los niños y niñas soportes culturales a dichas creencias o, que queden librados a sus propias fantasías. En nuestra cultura occidental, es un ejemplo el caso de la creencia en Papá Noel, en los Reyes magos y sustitutos según región) ${ }^{3}$.

Consideraremos a la patologización de la infancia como una alienante forma de tratamiento social de las diferencias. Nueva forma de expulsión de la sexualidad infantil del terreno de la educación y transmisión humana en la cultura.

Tanto desde el campo psicoanalítico (Freud, 1908a; 1908b; Klein, 1974; Bettelheim, 1999; Mannoni, 1990; Dolto, 1981; Lacan, 1994; entre otros) como desde los enlaces entre Infancia, Historia, Literatura y Filosofía (Agambem, 2003; Kohan, 2004; Lyotard, 1997; Delgado Criado, 2000) se considera de alto valor la posibilidad de los niños de formular preguntas ontológicas, en torno al nacimiento, al sexo, a Dios, a la muerte. La repercusión de estas preguntas en los mayores, no es sin consecuencias para los infantiles sujetos.

Por información relevada por Minnicelli (2011), en 100 casos de entrevistas iniciales administradas a padres al momento de ingreso al jardín de infantes, escuelas privadas de la ciudad de Mar del Plata, Argentina, sus respuestas fueron que "[...] no habían preguntado nada [...]" y que “[...] no sabrían responderles si los chicos les llegaran a preguntar".

Nuestra investigación clínica y clínico-social, nos permite sostener como hipótesis que la oferta de narrativas facilita la posibilidad de 
Tratamiento Social de las Diferencias, Teorias Infantiles y Narrativas

que los niños puedan construir puentes discursivos entre preguntas de difícil formulación y sus propias teorías en la medida que cuenten con recursos de anclaje imaginario promotores de alternativas de simbolización: relatos míticos, fantásticos, fábulas y leyendas. A sabiendas que dichas preguntas, no tendrán jamás respuestas unívocas, en esta dirección, las cuestiones de género cobran relevancia, ya que se incorporan otros imaginarios, otros ideales a los antiguos que confundían sexualidad con una única modalidad de respuesta: macho y hembra.

Considerando que la infancia es un momento lógico de constitución subjetiva, debemos detenernos en el auge que ha tenido en los últimos tiempos la patologización y medicalización de las vicisitudes propias a la misma.

Siguiendo a Michel Foucault (2000) podemos decir que la intrusión de la medicina en el terreno de la infancia se apoya en los siguientes factores:

- El trato que se da a la consideración de la sexualidad autoerótica infantil.

- Una concepción de inmadurez del desarrollo que concluye en diagnósticos de infantilismo.

- La importancia otorgada a la herencia biológica.

- La sociedad, atribuyéndose la función de protección social.

Esto podemos observarlo en el uso extendido del modelo psicopatológico que propone el Manuale Diagnostico e Statistico IV (DSM IV), minando el campo de la infancia con rótulos tales como Trastornos Generalizados del Desarrollo (TGD), Déficit Atencional con o sin Hiperactividad (ADD/ADH), Niños Oposicionistas y Desafiantes (ODD), Trastornos Obsesivos Compulsivos (TOC), Bipolares Infantiles (TBPI), entre otros.

La clausura de lo infantil en categorías diagnósticas y consecuentemente la aplicación de tratamientos ad hoc de uso universal, en definitiva, termina siendo excluyente de su propia infancia (Terzaghi, 2011).

Cuando hacemos referencia a la medicalización y la patologización tanto de la infancia como de la adolescencia, no es nuestra intención cuestionar de manera generalizada el avance de los conocimientos médicos que posibilitan la detección temprana de enfermedades; ni a las intervenciones terapéuticas que requieren el uso de medicación; así como tampoco al desarrollo científico tecnológico de la industria farmacéutica gracias a la cual se ha logrado mejorar de modo significativo la calidad de vida de amplios sectores de la población (Dueñas, 2011).

Sólo intentamos poner de relieve cierta tendencia que solemos encontrar en aquellos espacios donde transitan niños, niñas y adolescentes: pareciera que hoy, todo modo de transmisión se comprende mejor si es genético, orgánico o celular, es decir, si tiene un soporte material. Entonces, la mayoría de las veces, los adultos referentes parecen preferir ubicar la causa del trastorno que el niño puede padecer en algún aspec-

548 Educação \& Realidade, Porto Alegre, v. 38, n. 2, p. 539-554, abr./jun. 2013. Disponível em: <http://www.ufrgs.br/edu_realidade> 
to fisiológico, antes que en la trama del lenguaje que lo esperaba desde antes de nacer, y que luego lo alojó - o no - (L’Heuillet, 2008).

En esta misma dirección, transitan las cuestiones de género cuando se las vincula a lo estrictamente basado en definiciones anatómicas. Acordamos con Juan Vasen (2011) en que debe tratarse de que en los intervalos de los saberes escritos, otras verdades puedan hablar de lo impensado de ese pensamiento.

\section{Tal como afirma Silvia Bleichmar (2011):}

La concepción dominante, la más extendida, partía de una premisa falsa, que consiste en considerar que la realidad es siempre mejor que la fantasía. Todos sabemos que no es así, que la fantasía es en muchos casos mejor cuando la realidad es insoportable, que la realidad no despeja los fantasmas [...] (p. 26).

En estos tiempos nos enfrentamos ante las improntas de un borramiento de la trama narrativa que evidencie continuidades y discontinuidades históricas en los modos de definir los asuntos humanos respecto de las nuevas generaciones.

Acordamos con el interrogante de Françoise Dolto:

¿Cuáles son, pues las condiciones necesarias y suficientes en el ambiente de un niño, para que los conflictos inherentes al desarrollo de cada ser humano puedan resolverse para aquél en forma sana, es decir, creadora; para que al momento decisivo del Edipo y su resolución en la recomposición de los afectos, de las identificaciones y de los deseos incestuosos, se abra paso una persona actuante y responsable; para que la angustia de castración ligada al complejo de Edipo desemboque en el abandono de las fantasías arcaicas o perversas, intrafamiliares, y conduzca al sujeto a su expresión en la vida social mixta y la vida cultural simbólica, aceptando sus leyes? (1981, p. 20).

Al decir de Lacan (1983):

Aquí reaparece lo que ya les señalé, a saber, que el inconsciente es el discurso del Otro. Este discurso del Otro no es el discurso del Otro abstracto, del otro en la díada, de mi correspondiente, ni siquiera simplemente de mi esclavo: es el discurso del circuito en el cual estoy integrado. Soy uno de sus eslabones. Es el discurso de mi padre, por ejemplo, en tanto que mi padre ha cometido faltas que estoy absolutamente condenado a reproducir [...] Estoy condenado a reproducirlas porque es preciso que retome el discurso que él me legó, no simplemente porque soy su hijo, sino porque la cadena del discurso no es cosa que alguien pueda detener, y yo estoy precisamente encargado de transmitirlo en su forma aberrante a algún otro. Tengo que plantearle a algún otro el problema de una situación vital con la que muy posiblemente él también va a toparse, de tal suerte que ese discurso forma un pequeño circuito

Educação \& Realidade, Porto Alegre, v. 38, n. 2, p. 539-554, abr./jun. 2013. 
Tratamiento Social de las Diferencias, Teorias Infantiles y Narrativas

en el que quedan asidos toda una familia, toda una camarilla, todo un bando toda una nación o la mitad del globo. Forma circular de una palabra que está justo en el límite del sentido y el sinsentido, que es problemática (p. 141).

Y es aquí donde nos encontramos con una dificultad: la ausencia de narrativa, y su contracara la importancia de recuperarla. Narración pensada como un intento de crear otras significaciones en la transmisión simbólica de los bienes culturales; crear un borde para eso de lo Real que se torna inasible.

Tal como nos dice Vasen (2000), la historia hace al niño, la historia se hace niño; los niños y las niñas hacen, como y cuando pueden, sus historias.

Esto puede significar que la narrativa puede que requiera ser entendida no sólo como la capacidad de contar historias, sino también como la recuperación de relatos que nos puedan permitir entender lo que nos pasa hoy, considerando sus raíces históricas y culturales, míticas, en tanto producciones del orden del discurso humano.

\section{Narrativas, Apuestas en Educación por la Transmisión}

A partir del aporte del Psicoanálisis con niños y, de las Experiencias de Intervenciones Institucionales Interdisciplinarias recogidas en el III Simposio Internacional de Infancia, educación y derechos de niños, niñas y adolescentes ${ }^{4}$ ubicamos una coincidencia que nos interesa indagar respecto de la relación que se infiere entre:

a) la falta de ocasión para que los niños puedan no sólo formular sus preguntas ontológicas - que pueden presentarse de modos muy diversos $-\mathrm{y}$ las instancias de juego que permitan su paulatina elaboración y,

b) el estado de inquietud, dis-atención e hiperactividad que - de modo excesivamente generalizado - se busca aplacar con medicación psiquiátrica.

Esta relación no puede considerarse directa, es decir, no es posible en ningún asunto humano establecer causa-efecto, pero sí aspiramos a dar pruebas de cómo impacta en niños que expresan dicha sintomatología, "ceremonias mínimas" (Minnicelli, 2010) que le ofrecen posibilidades de expresión a las inquietudes que la sexualidad, nacimiento, Dios y muerte les provocan, remitiéndose en numerosos casos los síntomas cuando hay un escenario propiciatorio de la circulación de dichas inquietudes.

Las formas de tramitar la sexualidad es - y ha sido - legislada culturalmente desde tiempos remotos. Sea que dichas bases las ofrezca el discurso religioso o científico, sea que el marco lo ofrezcan los rituales culturales, en diferentes tiempos históricos ha encontrado marcos de referencia social. Hoy asistimos a un fenómeno que ofrece la posibilidad de que sea el discurso mercantil el que delimite lo permitido y lo 
prohibido respecto de los cuerpos; también en este sentido ingresan debates respecto del género, del sexo y de la sexualidad.

La importancia de revisar estos postulados es de alta significatividad ante la necesariedad de configurar marcos teórico clínico-sociales actualizados que contribuyan al sostén del reconocimiento de las diferencias, ante tendencias que desacreditan el impacto que presenta en los niños la exposición temprana a escenas de sexo sea mediante la imagen, sea que se los utilicen como objeto de goce voyeur adulto.

Diversas experiencias clínico-sociales, demuestran que la posibilidad de ofrecer escenarios socio-educativos - por ceremonias mínimas - donde la sexualidad encuentre cauces simbólico-imaginarios para dar borde a lo real, ubica a esos niños hiperactivos y desatentos en otra posición.

Así como debe establecerse la diferencia entre la lógica de las creencias - sustentada en la desmentida - respecto del alimento de la imaginación a través de relatos fantásticos, míticos, legendarios, cuentos de hadas, entre otros; debe establecerse la distinción fundante entre represión de la sexualidad que caracterizó a la época victoriana - en el sentido de represión social, por intolerancia a las diferencias de género - y represión de la sexualidad en el sentido de empuje al sexo, precipitación al sexo a la cual se tiende a arrojar a las nuevas generaciones.

Ya Foucault asimiló esta suerte de empuje al sexo, a hablar de sexo al dispositivo de la confesión.

La sexualidad infantil no puede ser hablada sino por alusión. No se trata de que sea educada por información sexual científicamente validada; se trata de generar espacios colectivos donde la sexualidad se manifieste en sus diferentes variantes; donde la sexualidad en el sentido de Eros aliente la creación de lazo social y de producción de cultura; sexualidad que encuentre condiciones de posibilidad para la instalación de diques psíquicos que enmarquen el empuje pulsional sin cauce psíquico.

Las vidas incipientes que se suponen sin historia, sin narrativas, sin juego, nos recuerdan que hace falta recuperar de nuestros antepasados la tenacidad con la que sostenían la diferencia entre lo inaccesible del territorio mítico y sus múltiples formas de representación, para dar continuidad a lo humano en el mundo por la transmisión y la memoria, a fin de sepultar los espectros de la historia y mantener con vida la producción de escrituras de la ley en la cultura.

Recebido em 11 de junho de 2012 Aprovado em 10 de setembro de 2012

\section{Notas}

1 Distinción planteada por MOREL, Geneviève. Ambigüedades Sexuales: sexuación y psicosis. Buenos Aires: Manantial, 2002, recuperada por CEVASCO, Rithèe. La discordancia de los sexos, Barcelona: S\&P, 2010

Educação \& Realidade, Porto Alegre, v. 38, n. 2, p. 539-554, abr./jun. 2013.

Disponível em: <http://www.ufrgs.br/edu_realidade> 
Tratamiento Social de las Diferencias, Teorias Infantiles y Narrativas

2 Ver Lajonquière (2000a; 2000b).

3 Para ampliar el tema, ver Minnicelli, 2010.

4 Las prácticas profesionales en los límites de la experiencia y del saber disciplinar, 11-12-13 de agosto de 2011. UNMDP- Facultad de Psicología. (Infancias y adolescencias: la construcción de sujeto (s) como premisa. de los Reyes, M. C. - (INFEIES-DiPro, Psicología, UNMDP; Escuelas de Jornada Extendida. Oportunidad para pensar nuevas maneras de sostener la responsabilidad pública ante las nuevas generaciones. Cardinale, L. M. Universidad Nacional del Comahue; El descuido del Otro, cuando los niños no nacen de un repollo. Castillo, M. C. Equipos de Orientación Escolar en GCBA; Una experiencia que anuda sentidos: Intervenciones psicoeducativas, subjetivación y emancipación. Alegre, S., Benedetti, E. UBA; ¿Falamos com as crianças? - Reflexões sobre as demandas dos educadores das creches. Mileib, T., Chaves, J., Oliveira Simone Pinto, M., Mesquita, R., Leite, C. FACED/FADIV - Faculdades de Ciências Econômicas, Administrativas e Contábeis de Divinópolis; Transitando por la categoría subjetividades juveniles: diversas formas de nombrarla, pensarla y habitarla. Bolletta, V, Castillo, A. CURZA-Viedma Universidad Nacional del Comahue; Los laberintos de la violencia infantil: La entrada es la misma que la salida. Chiani, L., Iglesias, M. UNLP; Infancias en contextos de vulnerabilidad: espacios de reflexión y juego como escenario de operaciones de subjetivación. Paolicchi, G., Colombres, R., Garau, A. Facultad de Psicología - UBA; Preocupaciones en torno a prescripciones y marcos normativos en el declive de las instituciones y la caída de la ley en la constitución de las subjetividades. Kundt, I.- FLACSO; Os problemas da infância e o mal-estar docente. Diniz M.- ICHS - UFOP; Un adecuado modelo profesional para la niñez de hoy, Nuñez, E.; A descoberta do nome: o próprio na infância e na escola. De Oliveira Leite, C.- UNICAMP; Relatos para no naufragar. Mendez, M., Centro de Atención Primaria de la Salud. Municipalidad de General Alvarado; Infancias y Políticas Públicas. Recrear (nos) en la práctica, hacer lugar a una clínica psicoanalítica. Alarcón, M., Baccelli, F., Codina, L., Crisci, M. S., Tazzo, M., Instituto de la Familia - Asoc.Civil sin fines de lucro - (Clínica psicoanalítica con niños, adolescentes y adultos) - Atención y Formación; Relatos de algunas experiencias subjetivantes en Club de Jóvenes y de Chicos por las sendas de las artes y de la lectura, la escritura y la narración oral. González Gainza, R. CAI - GCBA; Hablando de Dibujos Animados: iy si escuchamos a los niños?. Barbabella, M., Navarro, M., Galván, S. Universidad Nacional del Comahue; Recuperando la construcción de la ciudadanía en la infancia, Albarello, L., Rímoli, M., Spinello, A.-UNICEN; Aprender a Demorarse. Experiencias en dispositivos de intervención en niños y adolescentes. Benassi, M. J., Fraile, M. E. - UNL; Estilo é o que se transmite: uma leitura psicanalítica sobre a ação educativa. Monteiro, E. Faculdade de Educação da Universidade de São Paulo) entre otras.

\section{Referencias}

AGAMBEN, Giorgio. Infancia e Historia. Buenos Aires: Adriana Hidalgo, 2003.

BENÍTEZ, Mirtha; PERNICONE, Ariel. Fobias en la Infancia: de la historia biográfica de la familia Graf a la fobia en el discurso del psicoanálisis. Buenos Aires. Letra Viva, 2010.

BETTELHEIM, Bruno. Psicoanálisis de los Cuentos de Hadas. Barcelona: VEGAP, 1999.

BLEICHMAR, Silvia. Nuevas Condiciones de Producción de Patología en la In- 
fancia. In: DUEÑAS, Gabriela (Org.). La Patologización de la Infancia ¿Niños o Síndromes? Buenos Aires: Noveduc, 2011.

CEVASCO, Rithee. La Discordancia de los Sexos: perspectivas psicoanalíticas para un debate actual. Barcelona: $S \& P, 2010$.

DELGADO CRIADO, Buenaventura. Historia de la Infancia. Barcelona: Ariel, 2000.

DOLTO, Françoise. La Dificultad de Vivir. Buenos Aires: Gedisa, 1981.

DUEÑAS, Gabriela (Org.). La Patologización de la Infancia ¿̇Niños o Síndromes? Buenos Aires: Noveduc, 2011.

FERENCZI, Sandor. Confusión de Lengua entre los Adultos y el Niño: el lenguaje de la ternura y el de la pasión. In: XII CONGRESSO INTERNACIONAL DE PSICOANÁLISIS. Anais/Resumos... Wiesbaden, Alemanha, 1932.

FOUCAULT, Michel. Los Anormales. Buenos Aires: Fondo de Cultura Económica, 2000.

FREUD, Sigmund. Teorías Sexuales Infantiles. In: FREUD, Sigmund. Obras Completas. Madrid: Biblioteca Nueva, [1908a]. Volume 2.

FREUD, Sigmund. El Carácter y el Erotismo Anal. In: FREUD, Sigmund. Obras Completas. Madrid: Biblioteca Nueva, [1908b]. Volume 2.

FREUD, Sigmund. Introducción al Narcisismo. In: FREUD, Sigmund. Obras Completas. Madrid: Biblioteca Nueva, [1914]. Volume 2.

FREUD, Sigmund. Las Pulsiones y Sus Destinos. In: FREUD, Sigmund. Obras Completas. Madrid: Biblioteca Nueva, [1915]. Volume 2.

FREUD, Sigmund. Organización Genital Infantil: adición a la teoría sexual. In: FREUD, Sigmund. Obras Completas. Madrid: Biblioteca Nueva, [1923]. Volume 3 .

FREUD, Sigmund. La Disolución del Complejo de Edipo. In: FREUD, Sigmund. Obras Completas. Madrid: Editorial Biblioteca Nueva, [1924]. Volume 3.

FREUD, Sigmund. Algunas Consecuencias Psíquicas de la Diferencia Sexual Anatómica. In: Obras Completas. Madrid: Editorial Biblioteca Nueva [1925]. Volume 3.

JANIN, Beatriz. La Construcción de la Subjetividad: entre la Violencia y la Esperanza. In: DUEÑAS, Gabriela (Org.). La Patologización de la Infancia ¿2iños o Síndromes? Buenos Aires: Noveduc, 2011.

KLEIN, Melanie. El Psicoanálisis de Niños. Buenos Aires: Hormé, 1974.

KOHAN, Walter. Infancia entre Educación y Filosofía. Barcelona: Alertes, 2004.

LACAN, Jacques. El Seminario: libro 2, el yo en la teoría de Freud y en la teoría psicoanalítica. Buenos Aires: Paidós, 1983.

LACAN, Jacques. El Seminario: Libro 4: la relación de objeto. Buenos Aires: Paidós, 1994

LAJONQUIÈRE, Leandro. Infancia e Ilusión (Psico)Pedagógica. Buenos Aires: Nueva Visión, 2000a.

LAJONQUIÈRE, Leandro. O que da Infância a Ilusão (Psico)Pedagógica Mascara. Estilos da Clínica. Revista sobre a infância com problemas, São Paulo, Instituto de Psicologia da Universidade de São Paulo, v. 5, n. 8, p. 183-189, $2000 b$.

L’HEUILLET, Helene. El Psicoanálisis es un Humanismo. Buenos Aires: Letra Viva, 2008.

Educação \& Realidade, Porto Alegre, v. 38, n. 2, p. 539-554, abr./jun. 2013. 
Tratamiento Social de las Diferencias, Teorias Infantiles y Narrativas

LYOTARD, Jean-François. Lecturas de Infancia. Buenos Aires: Eudeba, 1997.

MANNONI, Maud. La Educación Imposible. México: Siglo XXI, 1990.

MINNICELLI, Mercedes. Infancias en Estado de Excepción. Derechos del niño y psicoanálisis. Buenos Aires: Noveduc, 2010.

MINNICELLI, Mercedes. Investigación realizada en el período Marzo-Julio, en el jardín de infantes Inti-Huasi, DIPREGEP 3009, Mar del Plata, Buenos Aires, Argentina, 2011.

PEUSNER, Pablo. Fundamentos de la Clínica Psicoanalítica Lacaniana con Niños: de la interpretación a la transferencia. Buenos Aires: Letra Viva, 2006.

TERZAGHI, María. Los Niños, los Médicos, la Medicalización. In: DUEÑAS, Gabriela (Org.). La Patologización de la Infancia iNiños o Síndromes? Buenos Aires: Noveduc, 2011.

VASEN, Juan. ¿Post-mocositos? Presencias, Fantasmas y Duendes en la Clínica con Niños y Jóvenes de Hoy. Buenos Aires: Lugar editorial, 2000.

VASEN, Juan. La Biblia y el Calefón. In: DUEÑAS, Gabriela (Org.). La Patologización de la Infancia ¿Niños o Síndromes? Buenos Aires: Noveduc, 2011.

Rosana Maneiro es psicoanalista Especialista en Infancia e Institución(es). Maestrando en Psicoanálisis. Investigadora Proyecto INFEIES: Estudio y análisis crítico del dispositivo integral de protección a la niñez y adolescência. Facultad de Psicología UNMDP, Buenos Aires, Argentina.

E-mail: rosmaneiro@copetel.com.ar

Mercedes Minnicelli es psiconalista Dra. en Psicología. Investigadora y Profesora. Facultad de Psicología UNMDP, Buenos Aires, Argentina. Directora red Interuniversitaria Internacional de estudios e investigaciones en Infancia e Institución(es) - INFEIES. Directora Proyecto de Investigación: INFEIES: Estudio y análisis crítico del dispositivo integral de protección a la niñez y adolescência.

E-mail: mercedes_minnicelli@hotmail.com 